\title{
Experimental investigation of the solid-liquid separation in a stirred tank owing to viscoelasticity
}

\author{
Weheliye Hashi Weheliye $\odot$, Giovanni Meridiano $\odot$, Luca Mazzei $\odot,{ }^{*}$ and Panagiota Angeli $\odot *$ \\ ThAMeS Multiphase, Department of Chemical Engineering, University College London, \\ Torrington Place, London WC1E 7JE, United Kingdom
}

(Received 8 January 2020; accepted 2 June 2020; published 29 June 2020)

\begin{abstract}
In this study, we investigated the motion of solid particles dispersed in highly viscous complex fluids agitated in a stirred vessel. We used a refractive index matching method and a combination of planar laser-induced fluorescence (PLIF), particle image velocimetry (PIV), and particle-tracking velocimetry (PTV) techniques to measure the velocity fields of the solid and fluid phases simultaneously along with the spatiotemporal distribution of the solids in the tank. The experimental data show that in a Newtonian ambient fluid, particles disperse uniformly in the plane of measurement, while in a strongly shear-thinning viscoelastic ambient fluid they tend to accumulate in the core of the vortices formed in the flow domain. We found that the solids migrate to the core of the vortices also when the ambient fluid is a Boger fluid, i.e., viscoelastic but not shear thinning. The effect of the first normal stress difference, $N 1$, on the vortex sizes and circulation intensities was also examined, with both properties decreasing for increasing $N 1$. Finally, we observed that the clustering time of the solids in the vessel for viscoelasticity-induced migration was at least three orders of magnitude lower than that obtained from the literature for inertia-induced migration.
\end{abstract}

DOI: 10.1103/PhysRevFluids.5.063302

\section{INTRODUCTION}

Mixing of solids in liquids is a fundamental unit operation in process engineering, where homogenous solid-liquid suspensions are often required to manufacture the desired product. Efficient fluid-particle mixing is necessary in several processes, such as those involving solid-catalyzed reactions, the manufacturing of formulations and the dissolution of solid additives. Mechanically agitated tanks are widely used owing to their versatility, comparative simplicity, and effectiveness in providing fast mixing [1].

Most of the studies on mechanically agitated fluid-particle mixers have focused on turbulent Newtonian flows, even if many industries (e.g., formulation, food, and energy) make significant use of highly viscous Newtonian and non-Newtonian fluids, which are notoriously troublesome to mix [2]. These studies have considered both $\mathrm{mm}$ - and $\mu \mathrm{m}$-size particles, observing different effects on the fluid dynamics. At the mm scale, Gabriele et al. [3] evaluated the effect of solid concentration (1\%-5\% by volume fraction) for particles of $1.5 \mathrm{~mm}$ diameter on the fluid mean velocity and turbulent kinetic energy (TKE) distributions in a vessel agitated by a pitched blade turbine (PBT), finding that the former did not vary with increasing solids content. The turbulent kinetic energy, conversely, significantly decreased, with the root mean square (rms) velocity decreasing by up to 40\%. Studies involving $\mu \mathrm{m}$-size particles are more numerous [4-8]. Micheletti and Yianneskis

*Corresponding authors: 1.mazzei@ucl.ac.uk; p.angeli@ucl.ac.uk 
[7] investigated the flow of suspensions of particles of $186 \mu \mathrm{m}$ diameter in a vessel agitated by a Rushton turbine, considering volumetric concentrations of up to $2 \%$. They observed that the particles suppressed both the mean and the rms of the radial fluid velocity component in the impeller plane, with the rms of the radial fluid velocity component decreasing by around $50 \%$ when compared with single-phase values.

The few studies on the laminar mixing of fluid-particle suspensions involved Newtonian fluids and aimed to evaluate how the configuration of the mixing device (e.g., shape and dimensions of impellers, presence of baffles), the properties of the phases and the operating conditions influence the system performance [9-13]. For example, Ibrahim and Nienow [10] investigated the effect of the impeller shape on the value of the minimum impeller speed needed to suspend the solid phase (i.e., the "just-suspending speed," $N_{j s}$ ) for a large range of Reynolds numbers, while Lassaigne et al. [11] adopted a PBT to show that the $N_{j s}$ increases when the particle diameter, the solid volume fraction, or the liquid viscosity increase. Limited information is available on the solid-liquid mixing in viscoelastic fluids. To the authors' knowledge, the few existing studies are limited to the critical impeller speed required for particle drawdown and to the liquid phase mixing times $[14,15]$.

In most of the studies reported above, the velocity fields were measured with laser-based techniques, such as particle image velocimetry (PIV) [3,8,16,17] and laser Doppler anemometry (LDA) $[5,7,18]$. When these techniques are applied to solid-liquid systems, especially dense ones, optical accessibility is a major limiting factor. Particles obstruct and scatter the laser light, which leads to results of poor quality. This is why the solid volume fraction in some of the studies mentioned above was limited to $1 \%[5,8,17]$. To overcome this problem, researchers have proposed to match the refractive index between the solids and the surrounding liquid $[3,7,13,16,18,19]$. The study by Gong et al. [13] was the first to match the refractive indices of the solid and fluid phases in a laminar flow, comparing the mean fluid velocities obtained from experiments with those found numerically via CFD simulations.

A phenomenon recently encountered when dealing with solid-liquid suspensions in stirred vessels is the spontaneous clustering of the solid phase. The involuntary accumulation of solids in separate regions of the flow represents a clear impairment to the mixing process and can affect negatively the quality of the products and/or the efficiency of the whole operation. The study by Wang et al. [20] examined solid-liquid suspensions in Newtonian and non-Newtonian laminar flows, adopting a three-dimensional (3D) particle visualization method to expose the real-time trajectories of the particles. In their study, they showed that for particle Stokes number much less than unity, the particles clustered in the cores of the toroidal vortices created by the impeller rotation. In particular, the revolving blades of the impeller generated two coexisting, confined regions, above and below the blades, wherein the particles migrated. The migration was induced by inertial perturbations in the flow outside these regions, where, owing to the high shear rates present near the blades, the particle trajectories deviate from the path lines of the fluid, enabling the particles to cross the boundaries of these regions and settle into their cores.

In this work, we investigated the motion of solid particles dispersed in highly viscous complex (that is, viscoelastic) fluids agitated in a stirred vessel, focusing on the clustering phenomenon, a process we attempted to explain. This work had two aims: to understand better how viscoelastic fluids affect solid mixing in stirred vessels and to offer an alternative and effective method for separating particles from highly viscous liquids, without any dilution, by making the particles cluster into specific regions of the flow domain. To this end, we used a refractive index matching method and a combination of PLIF and PIV/PTV techniques to measure the velocity fields of the solid and fluid phases simultaneously. We considered a strongly shear-thinning viscoelastic fluid and two viscoelastic fluids with constant viscosity (i.e., Boger fluids) to isolate the effects of elasticity from those of shear thinning. 
TABLE I. Properties of the fluids measured at $23^{\circ} \mathrm{C}$.

\begin{tabular}{|c|c|c|c|c|c|c|c|c|c|c|}
\hline Acronym & $\begin{array}{c}\text { Glycerol } \\
\text { [wt } \%]\end{array}$ & $\begin{array}{l}\text { Water } \\
\text { [wt\%] }\end{array}$ & $\begin{array}{c}\text { Zinc chloride } \\
\text { [wt\%] }\end{array}$ & $\begin{array}{l}\text { Xanthan } \\
\text { gum [ppm] }\end{array}$ & $\begin{array}{c}\text { PAA } \\
\text { [ppm] }\end{array}$ & $\begin{array}{c}\rho \\
{\left[\mathrm{kg} / \mathrm{m}^{3}\right]}\end{array}$ & $\begin{array}{c}\mu \\
{[\mathrm{Pa} \mathrm{s}]}\end{array}$ & $r_{n}$ & Rheology & $\operatorname{Re}$ \\
\hline GWZ-N & 42 & 18 & 40 & - & - & 1630 & 0.25 & 1.487 & Newtonian & 65,100 \\
\hline GWZ-NN & 42 & 18 & 40 & 1300 & - & 1630 & $5.9-0.27$ & 1.487 & Non-Newtonian & 65,100 \\
\hline GW & 98 & 2 & - & - & - & 1250 & $\approx 0.71$ & 1.475 & Newtonian & $33-50$ \\
\hline GW-B1 & 98 & 2 & - & - & 100 & 1250 & $\approx 0.8$ & 1.475 & Boger fluid & $33-50$ \\
\hline GW-B2 & 98 & 2 & - & - & 200 & 1250 & $\approx 0.85$ & 1.475 & Boger fluid & $33-50$ \\
\hline PMMA & - & - & - & - & - & 1190 & - & 1.487 & - & - \\
\hline
\end{tabular}

\section{EXPERIMENTAL SETUP}

\section{A. Rheology}

In this study, we investigated five fluids, whose compositions and rheological parameters are given in Table I. The rheological curves of these fluids were obtained by a cone and plate measuring system in a rotational rheometer (Anton Paar Physica MCR 302). All the experiments were carried out in a temperature-controlled laboratory at $23^{\circ} \mathrm{C}$ to within $\pm 1{ }^{\circ} \mathrm{C}$, and so all the rheological measurements were conducted at this temperature. The laboratory temperature was stable enough that no further temperature control on either the apparatus or the fluids was necessary. The first fluid (GWZ-N) was a Newtonian mixture of glycerol (42\% by weight), water (18\%), and zinc chloride (40\%), which resulted in a viscosity of $0.25 \mathrm{~Pa}$. The second fluid (GWZ-NN) consisted of the same mixture as the Newtonian fluid GWZ-N with the addition of $1300 \mathrm{ppm}$ of xanthan gum, a compound that rendered it strongly shear thinning. The mixtures were chosen to match the refractive index of polymethylmethacrylate (PMMA) solid particles, which were used to generate the suspensions. To fit the rheological data of the non-Newtonian GWZ-NN mixture, we used the Carreau-Yasuda model (C-Y model; see Fig. 1):

$$
\mu_{a}=\mu_{\infty}+\left(\mu_{0}-\mu_{\infty}\right)\left[1+\left(\lambda_{C Y} \dot{\gamma}\right)^{2}\right]^{\frac{n-1}{2}},
$$

where $\mu_{a}$ is the apparent viscosity, $\mu_{\infty}$ is the viscosity on the plateau where the shear rate is very high (in the limit to infinity), $\mu_{0}$ is the viscosity on the plateau where the shear rate is very small (in the limit to zero), $\lambda_{C Y}$ is a time constant, $\dot{\gamma}$ is the shear rate (that is, the magnitude of twice the

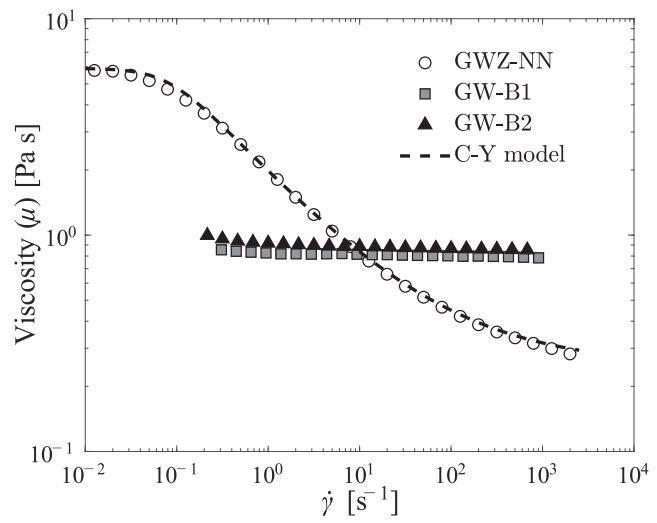

(a)



(b)

FIG. 1. (a) Viscosity and (b) first normal stress difference, $N 1$, as functions of the shear rate for mixtures GWZ-NN, GW-B1, and GW-B2. 
rate of deformation tensor) and $n$ is the shear index. As we can observe from Fig. 1(a), as the shear rate increases, the apparent viscosity of the mixture reduces by about an order of magnitude, with a shear index $n$ of 0.53 and a time constant $\lambda_{C Y}$ of $12.3 \mathrm{~s}$. The zero shear-rate viscosity $\left(\mu_{0}\right)$ and the infinite shear-rate viscosity $\left(\mu_{\infty}\right)$ are approximately equal to $5.9 \mathrm{~Pa} \mathrm{~s}$ and $0.25 \mathrm{~Pa}$, respectively. The $\mathrm{C}-\mathrm{Y}$ model fits the experimental data to within $2 \%$. The range of shear rates investigated varies from $0.01 \mathrm{~s}^{-1}$ to $2500 \mathrm{~s}^{-1}$, which is representative of the range encountered in stirred vessels [21]. Even though a small amount of xanthan gum $(1300 \mathrm{ppm})$ was present in the GWZ-NN mixture, the latter exhibited elastic behavior. This was quantified by the first normal stress difference, $N 1$, obtained during the acquisition of shear stress and shear rate data on the same instrument through normal force measurements. As Fig. 1(b) shows, for the GWZ-NN mixture the first normal stress difference increases with the shear rate.

To isolate the effects of elasticity from those of shear thinning, we prepared two Boger fluids, GW-B1 and GW-B2. To this end, we used a high-molecular-weight polyacrylamide (PAA), a compound that yields viscoelasticity even at very low concentrations [22]. Although the GWZ-N mixture matched the refractive index of the solid, we could not use it to generate the Boger fluids, because adding a small amount of PAA to the mixture resulted in small first normal stress differences, $N 1$, that could not be measured reliably, whilst increasing the PAA concentration led to shear-thinning behavior before any elasticity could be detected. For this reason, we used a Newtonian mixture (GW), made of glycerol (98\% by weight) and water $(2 \%)$, to which $100 \mathrm{ppm}$ and $200 \mathrm{ppm}$ of PAA $\left(M_{w}=5-6 \times 10^{6} \mathrm{~g} / \mathrm{mol}\right)$ were added to produce two Boger fluids. As Fig. 1(a) reveals, both Boger fluids possess constant viscosities. The elastic responses are shown in Fig. 1(b), where $N 1$ for both mixtures increases with the shear rate. At low shear rates, $N 1$ is proportional to $\dot{\gamma}^{2}$ (i.e., slope of 2 in the logarithmic plot), a response that is typical for "true" Boger fluids [22]. It can also be seen that over the whole measured range, GW-B1 displays lower values of $N 1$, and thus lower levels of elasticity.

\section{B. Selection of the solid particles}

To visualise the flow of the liquid phase in the presence of solids, we matched the refractive indices of the two phases. PMMA spherical particles of $1.5 \mathrm{~mm}$ diameter and refractive index of 1.487 were employed as solid dispersed phase. The refractive indices of both GWZ-N and GWZ-NN fluids, measured with a refractometer (Abbe 5, BS), were found to be equal to $1.487 \pm 0.0005$ (at $23^{\circ} \mathrm{C}$ ). The refractive indices were relatively insensitive to temperature (decreasing from 1.487 to 1.485 with a $5{ }^{\circ} \mathrm{C}$ temperature rise), so that no temperature correction was required. To avoid any overheating of the fluids, mainly caused by the rotating impeller, we kept the experimental runs short. The experiments were conducted at particle volumetric concentration ranging from 0 to $2 \%$, with impeller speeds $400 \leqslant N \leqslant 1000 \mathrm{rpm}$, so that the particles were always sufficiently suspended, the air-liquid interface remained essentially flat, and any image distortion was avoided.

For the Boger fluids, however, their refractive index $\left(1.475\right.$ at $\left.23{ }^{\circ} \mathrm{C}\right)$ differed from that of the solids. To avoid excessive scattering of the laser light, which would have lowered the quality of the images, we measured the velocity of the ambient fluid (via PIV) and the particles distribution separately. To suspend the particles sufficiently and maintain a flat air-liquid interface, we employed impeller speeds, $N=800-1500 \mathrm{rpm}$.

\section{Systems and flow geometry}

The experiments were carried out in a cylindrical flat-bottomed vessel of diameter $T=50 \mathrm{~mm}$, as shown in the schematic diagram of Figs. 2(a) and 2(b). The liquid in the vessel was agitated by a stainless steel dual impeller with two flat blades [see Fig. 2(a)] of diameter $D=37.5 \mathrm{~mm}$, blade height of $W=6 \mathrm{~mm}$, impeller clearance from the bottom of the vessel of $C=10 \mathrm{~mm}$, and a liquid height of $H=60 \mathrm{~mm}$. The vessel was encased in a square trough, filled with the fluid mixture GWZ-N in order to minimize the optical distortion resulting from the curvature of the cylindrical 




(a)

(b)

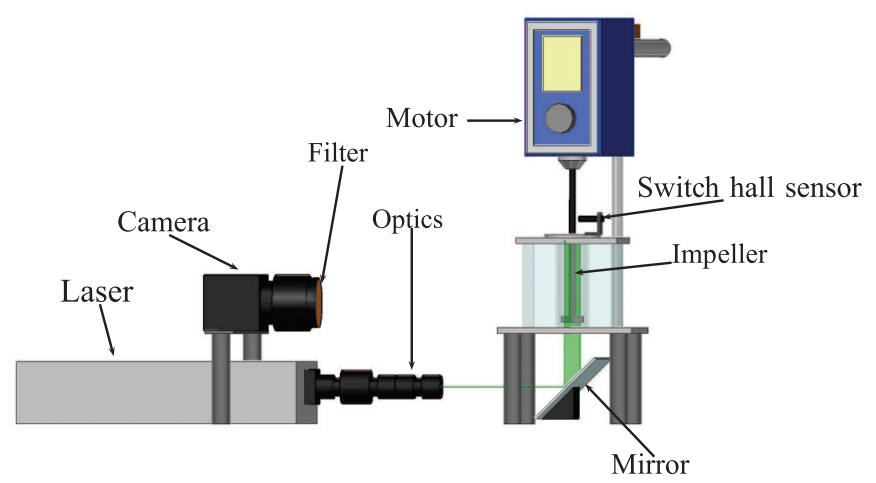

(c)

FIG. 2. (a) 3D visualizations of the dual blade impeller; (b) schematic of the tank geometry showing visualization areas for both PIV/PTV and solid mixing configurations; (c) experimental setup for the PIV.

surface. Both the vessel and the trough were made of acrylic (refractive index 1.495) to match the refractive index of the working fluid and of the dispersed phase (PMMA). At the beginning of each run, the particles were placed at the top of the liquid in the vessel. To avoid air entrainment when the agitation started, we placed a lid on top of the liquid surface, so that the floating particles were entirely wetted.

The Reynolds number, Re $\equiv \frac{\rho_{f} N D^{2}}{\mu}$, for the Newtonian (GWZ-N and GW) and Boger (GW-B1 and GW-B2) mixtures was based on the impeller diameter $D=37.5 \mathrm{~mm}$, impeller speed $N$, fluid density $\rho_{f}$, and constant viscosity $\mu$. For the shear-thinning fluid (GWZ-NN), the Reynolds number was defined according to the procedure of Metzner and Otto [23], which uses an average shear rate in the tank, $\langle\dot{\gamma}\rangle$, linearly related to the rotational speed of the impeller:

$$
\langle\dot{\gamma}\rangle=k_{s} N
$$

where the value of $k_{s}$ depends on the impeller type. The impeller used in our work was similar to that adopted by Cortada-Garcia et al. [21], for which $k_{s}=15$. Once $\langle\dot{\gamma}\rangle$ was estimated, we found the apparent viscosity from Eq. (1), which fitted the rheological data of GWZ-NN [Fig. 1(a)]. Thus, the Reynolds number for the GWZ-NN fluid was given by

$$
\operatorname{Re}=\frac{\rho_{f} N D^{2}}{\mu_{\infty}+\left(\mu_{0}-\mu_{\infty}\right)\left[1+\left(\lambda_{C Y} k_{s} N\right)^{2}\right]^{\frac{n-1}{2}}} .
$$


From the above, the average shear rates for the GWZ-NN mixture are $\langle\dot{\gamma}\rangle=175$ and $250 \mathrm{~s}^{-1}$ for $N=700 \mathrm{rpm}$ and $1000 \mathrm{rpm}$, respectively, which correspond to $\operatorname{Re}=65$ and $\operatorname{Re}=100$. Both values of $\langle\dot{\gamma}\rangle$ lie within the shear-thinning regime of GWZ-NN [Fig. 1(a)].

An elastic dimensionless number that will be considered below to quantify the role of fluid elasticity is the Weissenberg number, given by $\mathrm{Wi}=\lambda N$, where $\lambda$ is the elastic relaxation time of the fluid and $N$ is the impeller speed (in $\mathrm{rad} / \mathrm{s}$ ). The relaxation time of a dilute polymeric solution can be evaluated according to Zimm's theory from [24-26]

$$
\lambda=\frac{F[\eta] M_{w} \mu_{s}}{R T},
$$

where $R$ is the ideal gas constant, $T$ is the absolute temperature, $M_{w}$ is the molecular weight of the polymer, $\mu_{s}$ is the solvent viscosity, and $[\eta]$ is the intrinsic viscosity that can be estimated from the Mark-Houwink correlation, $[\eta]=0.0049 M_{w}^{0.8} \approx 1300 \mathrm{ml} / \mathrm{g}$, for the polyacrylamide (PAA) polymer in a water-glycerol mixture $[25,27,28]$. The prefactor $F$ is given by $1 / \sum_{i=1}^{\infty}\left(\frac{1}{i^{3 v}}\right) \approx 0.5313$, where $v$ is a parameter that depends on the quality of the solvent and is equal to 0.6 for polymers suspended in a good solvent [26]. The relaxation time for the ambient fluids GW-B1 and GW-B2 was found to be $\lambda \approx 1.1 \mathrm{~s}$. Equation (4) shows that the relaxation time $\lambda$ is independent of the polymer concentration; this is only valid when the polymer concentration $c$ is less than the overlapping concentration $c^{*}$ (i.e., the concentration at which the polymer chains start to interact) [26]. The overlapping concentration $c^{*}$ can be estimated from $c^{*}=\frac{1}{[\eta]}$ [29] and is $770 \mathrm{ppm}$ for the current system, higher than the polymer concentrations employed in this study.

\section{Particle image velocimetry measurements}

The PIV set-up included a dual cavity Nd:Yag green laser (532 nm) (Litron Laser, $14.5 \mathrm{~Hz}$, $1200 \mathrm{~mJ}$ ) and a straddling CCD camera with $2048 \times 2048$ pixels with a spatial resolution of $32 \mu \mathrm{m} / \mathrm{px}$, equipped with a $105 \mathrm{~mm}$ Nikon lens. As tracers for the PIV measurements, we used fluorescent polymer particles with $20 \mu \mathrm{m}$ diameter made of melamine resin and coated with Rhodamine $\mathrm{B}$, which at room temperature has a high fluorescent intensity. For the experimental conditions investigated, the tracer relaxation time was negligible compared to the convection time (St $=\frac{\rho_{p} d_{p}^{2}}{18 \mu} N \ll 1$, where $\rho_{p}$ and $d_{p}$ are the tracer density and diameter, respectively). To distinguish between the solid and the fluid phases when their refractive indices were matched, we added a small amount of aqueous Rhodamine $6 \mathrm{G}$ dye in the liquid. For the experiments with the Boger fluids, where the refractive indices were not matched, only the solid particles were dyed. We employed an orange filter with a cutoff wavelength at $570 \mathrm{~nm}$ to ensure that only the light emitted from the tracers and the particles (maximum emission at $590 \mathrm{~nm}$ ) was recorded by the camera. The laser and the camera were synchronized by means of a Laser Pulse Synchroniser (Model 610035 TSI) and they were controlled via the Insight 4G (TSI) software. The laser beam was guided through a collimator and two cylindrical lenses $(25 \mathrm{~mm}$ and $15 \mathrm{~mm})$ that transformed it into a narrow plane of $1 \mathrm{~mm}$ thickness.

Measurements were obtained on both the vertical and horizontal planes. For the vertical plane measurements, the generated laser sheet was reflected on a $45^{\circ}$ silver-coated mirror and entered the vessel from the bottom as shown in Fig. 2(c). For the measurements on the horizontal plane, a horizontal laser sheet was generated just above the top impeller while the camera was positioned underneath the vessel (not shown in the figure).

We captured the images using either phase-resolved or time-resolved measurements. For the former, the image capture was synchronized with the impeller blade position using a hall switch sensor. For each experiment, 100 image pairs were taken at the same phase angle (i.e., the angle between the upper blade of the impeller and the laser sheet) to produce 100 instantaneous velocity vector fields. The phase-resolved velocity field was then obtained by averaging the instantaneous vector fields. Phase-resolved experiments were carried out for PIV/PTV measurements and the time between each laser pulse $\Delta t$ varied depending on the speed of the impeller to ensure that the 
tracers did not move out of the image plane during capture. To maximise the field of view in the vertical plane, we carried out the phase-resolved measurements at an angle of $5^{\circ}$ to the top blade, as shown in the plane view on the right-hand side of Fig. 2(b), and only at the left-hand side of the vessel (blue box). In addition, we obtained horizontal plane vector fields through phase-locked measurements on a single plane at $z / T=0.8$. However, to calculate the shear rates, we measured PIV data on different horizontal planes, $z / T=0.78-0.82$, with intervals of $\Delta z / T=0.02$, along with two vertical PIV measurements from $\phi=5-7^{\circ}$ at an interval of $\Delta \phi=2^{\circ}$. For the same image magnification and spatial resolution, we carried out time-resolved measurements to investigate the distribution of the solids. We captured more than 1000 images in these measurements, with image acquisition frame rates of $14.5 \mathrm{~Hz}$, utilizing the whole field of view, as shown in Fig. 2(b) (red box).

\section{E. Image analysis}

Figure 3(a) shows a typical raw image obtained from the PIV measurements in the GWZ-NN mixture (1300 ppm xanthan gum) at $\mathrm{Re}=65$. The bright spots correspond to the tracers in the continuous phase, while the bright circles represent the suspended particles. Each pixel in the 8-bit images has an intensity value ranging from 0 (black) to 255 (white). Based on the intensity histogram of the raw image in Fig. 3(a), we used a threshold value to binarise the images and isolate the tracer particles and the edges of the solid spheres, as shown in Fig. 3(b). Subsequently, we applied a median filter to the binarized images to remove the tracer particles. To detect the edges of the particles, we used a circular Hough transform (CHT) algorithm [30]. According to the algorithm, at each edge point of a solid sphere, circles are drawn with center in the edge point considered and varying radii, $0.2<r<0.75 \mathrm{~mm}$. A range of radii is chosen because not all the solid spheres are placed centrally in the laser plane. Once every edge point and every desired radius had been used, we selected as sphere center the point passed by the highest number of circles. Figure 3(c) shows the boundaries detection for the particles in the image.

The images shown in Fig. 3(c) display the position of the particles, which were then used in particle tracking velocimetry (PTV) and solids mixing analysis. To track the velocities of the particles, we used the cross-correlation technique in the PTVlab toolbox by Brevis et al. [31], which involves tracking the motion of individual particles between two successive camera frames. First, each particle is detected and localized on each camera frame; then, the two particle images in the two consecutive frames are "matched," i.e., identified as belonging to the same particle without ambiguity. The difference between the positions of the two particle images gives the particle displacement between the two frames, while the time is given by the time difference between two consecutive images.

To quantify the degree of mixing of the particles in the tank over time, we employed a similar method to that developed by Camesasca et al. [32]. According to their approach, the measurement plane was split in small subregions, $i$, and the Shannon entropy, $S_{i}$, was calculated in each one as follows:

$$
S_{i}=-\sum_{j=1}^{2} p_{j}(i) \ln \left[p_{j}(i)\right],
$$

where $p_{1}(i)$ is the ratio of the number of pixels filled by the solids over the total number of pixels in region $i$ and $p_{2}(i)$ is the ratio of the number of pixels filled by the fluid over the total number of pixels in subregion $i$. The entropy for the whole field of view is then estimated as the average of the entropies of all the subregions:

$$
S=\frac{1}{N_{\text {regions }}} \sum_{i=1}^{N_{\text {regions }}} S_{i} .
$$

The entropy, $S$, of mixing is defined as the increase of disorder in a solid-liquid system upon transition from a fully demixed to an ideally mixed state [33]. The entropy of a hypothetically 




(a)

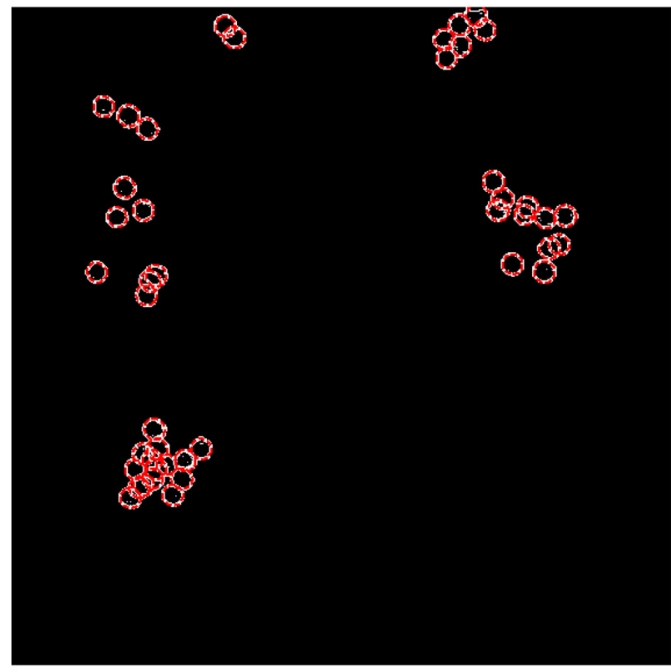

(c)

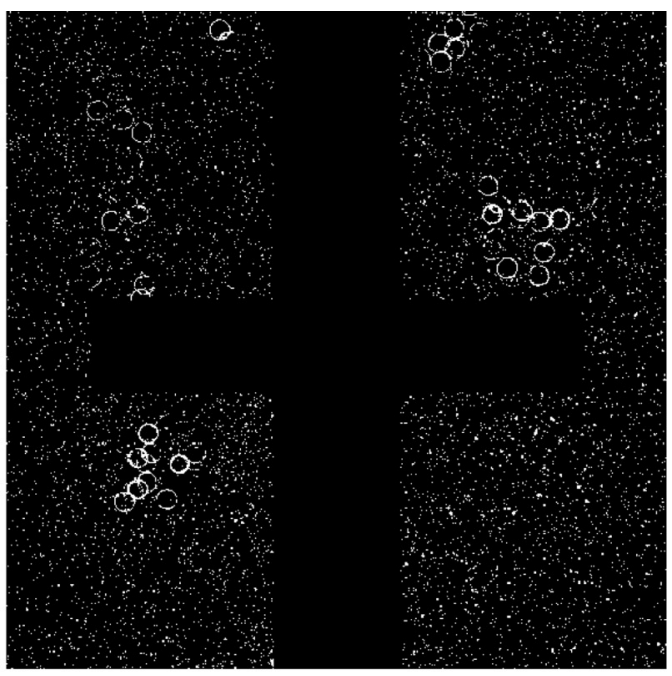

(b)

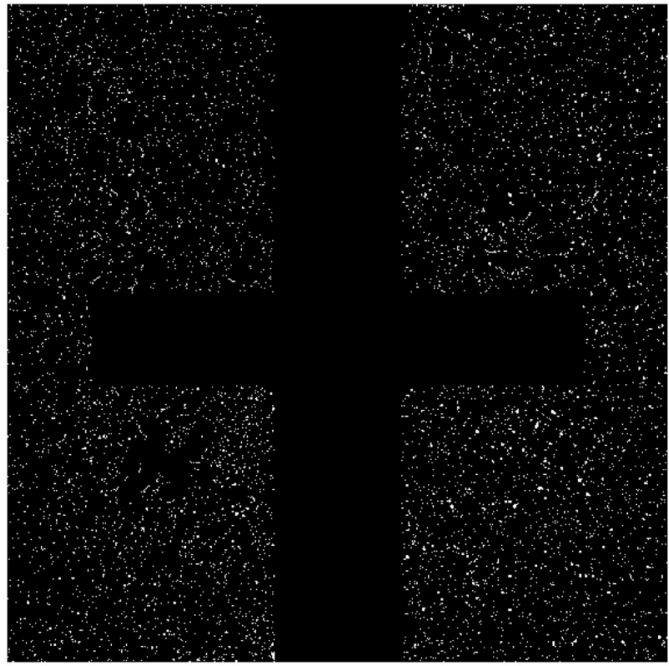

(d)

FIG. 3. Image analysis during pretreatment stages, for $\mathrm{Re}=65, \mathrm{GWZ}-\mathrm{NN}$ mixture and particle volumetric concentration of $1 \%$, where (a) raw PIV image; (b) binarized image without the dye; (c) image with the detected solids; (d) image with only the PIV tracer particles.

perfectly mixed system (for which the particles are uniformly distributed across the field of view) is $S=1$. The "worst" mixing scenario, corresponding to the state of the system before stirring has started, when all the particles are located at the top of the liquid, is characterized by a low value of $S$.

By subtracting Fig. 3(c) from Fig. 3(b), one can isolate the signal of the tracers, as shown in Fig. 3(d). These images were then binarized to eliminate the effect of the dye. We calculated the velocity of the fluid from the tracer displacements between two consecutive images with the freeware package JPIV, using a 50\% window overlap for a final interrogation window with resolution of $16 \times 16$ pixels. An amplitude filter was applied to each cross-correlation box to eliminate the vectors that substantially deviated from the median value [34]. Since the solid spheres 


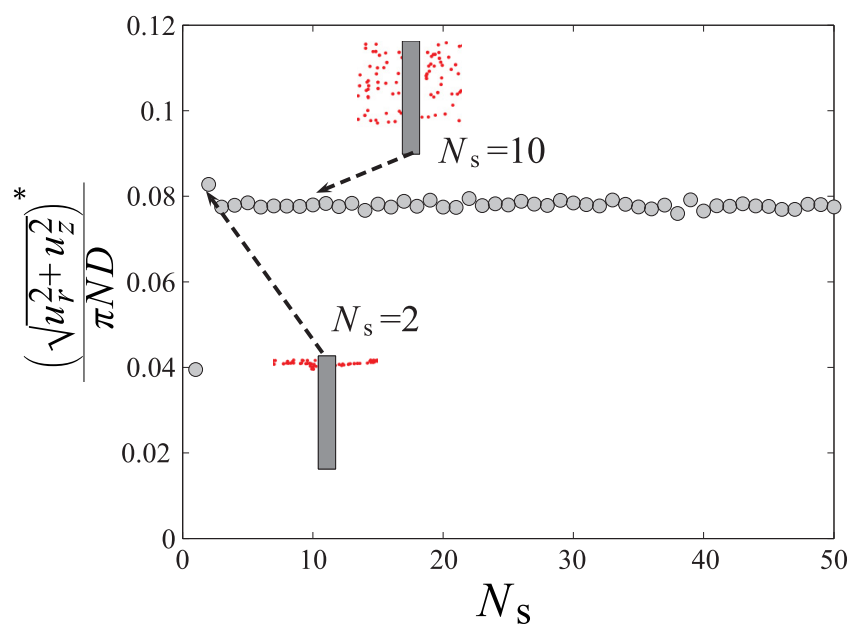

FIG. 4. Variation of the dimensionless mean fluid velocity magnitude, $\left(\sqrt{u_{r}^{2}+u_{z}^{2}}\right)^{*}$, as a function of the number of revolutions $N_{s}$ for the fluid mixture GWZ-N and $\mathrm{Re}=65$.

were larger than an individual interrogation window, this caused a larger number of zero values in the single phase. We omitted these values in calculating the phase-average velocity fields.

For the experiments with the Boger fluids, where the refractive indices of the fluids and the particles were not matched, we conducted the PIV measurements without solids to obtain the velocity fields in the liquid phase. The processing was the same as that described above. We then repeated the experiments with the solids present to obtain the time evolution of their mixing. For these measurements, the spheres were dyed with Rhodamine $6 \mathrm{G}$ and were tracked with the CHT algorithm described above.

\section{F. Steady-state nature of the flow}

To establish whether the fluid velocity field in the vessel was developed before the particles were drawn in, we investigated the temporal evolution of the flow field for the case of the GWZ-N fluid mixture. We calculated the spatial mean fluid velocity magnitude, $\left(\sqrt{u_{r}^{2}+u_{z}^{2}}\right)^{*}$, from the phaseresolved measurements previously described, using the following equation:

$$
\left(\sqrt{u_{r}^{2}+u_{z}^{2}}\right)^{*} \equiv \frac{\int_{A}\left(\sqrt{u_{r}^{2}+u_{z}^{2}}\right) d A}{A},
$$

where $u_{r}$ and $u_{z}$ are the radial and axial fluid velocity components, respectively, on the plane of measurement and $A$ denotes the area over which the integral is computed, in our case the left-hand side of the tank [blue area in Fig. 2(b)]. We calculated $\left(\sqrt{u_{r}^{2}+u_{z}^{2}}\right)^{*}$, at several times. Each of these times referred to the same position of the impeller blades [phase angle $\phi=5^{\circ}$; see Fig. 2(b)] but to a different number of revolutions from when the impeller was started. Hence, instead of reporting $\left(\sqrt{u_{r}^{2}+u_{z}^{2}}\right)^{*}$, as a function of time, here we report it as a function of the number of revolutions, $N_{s}$. Figure 4 reports the experimental results for $\mathrm{Re}=65$. These measurements were carried out in the presence of the solids. As we can see from Fig. 4, the mean fluid velocity sharply increases initially, but it settles to a nearly constant value after just three revolutions $\left(N_{s}=3\right)$. The solids are still located at the free surface for $N_{s}=2$ (see the inset of Fig. 4) indicating that the solids are only drawn in when the flow has reached a steady state. As we see from the inset of Fig. 4, at $N_{s}=10$ the solids are uniformly distributed over the upper part of the vessel. 




(a)

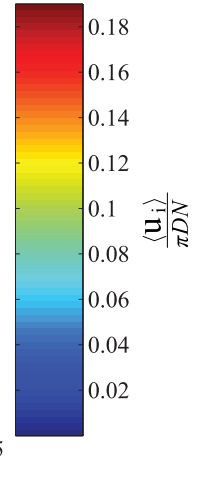

(1)

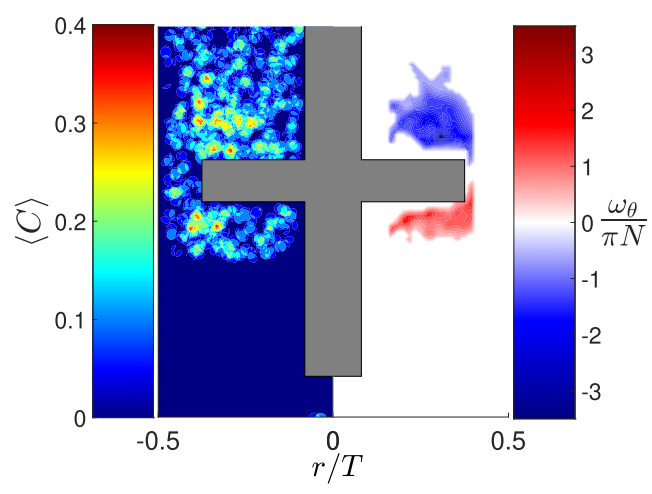

(b)

FIG. 5. (a) Phase-averaged fluid (left figure) and solid (right figure) velocity fields; (b) contour plots of the averaged solid volume fraction and dimensionless tangential component of the vorticity, $\omega_{\theta}$, of the upper part of the vessel for the fluid mixture GWZ-N at Re $=65$ and particle volumetric concentration of $1 \%$.

\section{RESULTS}

\section{A. Shear-thinning polymer solution}

We first examine the motion of the particles in the Newtonian mixture, GWZ-N, at steady state. Figure 5(a) shows the phase-averaged velocity fields of the fluid (left plot) and solid (right plot) phases at $\operatorname{Re}=65$. The phase-averaged velocity, $\left\langle\mathbf{u}_{i}\right\rangle$, is given by

$$
\left\langle\mathbf{u}_{i}\right\rangle=\frac{1}{N_{\phi}} \sum_{i=1}^{N_{\phi}} \mathbf{u}_{i},
$$

where $\mathbf{u}_{i}$ is the instantaneous velocity field and $N_{\phi}$ corresponds to the number of measurements taken at a phase angle, $\phi=5^{\circ}$. The velocity vectors have been normalized with the impeller tip speed. The flow field generated by the dual paddle impeller is similar to that reported in Cortada-Garcia et al. [21], with the fluid ejected from the impeller radially towards the tank wall. The fluid then moves axially and curves towards the center line of the tank, where it is drawn towards the impeller. All the fluid appears to move; no stagnant zones can be identified. Figure 5(a) (right plot) seems to indicate that the solid spheres follow the fluid pathlines in their vortical motion with the velocity of each sphere being close to the local velocity of the fluid. This is expected as inertia effects are negligible. For this case with density difference between the two phases equal to $440 \mathrm{~kg} \mathrm{~m}^{-3}$ the particle settling velocity due to gravity, $U_{t}$, is much less than the characteristic velocity of the fluid, $\pi N D\left(U_{t} / \pi N D \sim 1 \times 10^{-3} \ll 1\right)$. In addition, the particles Stokes number is less than unity (St $\sim 1 \times 10^{-3} \ll 1$ ), i.e., the particles relax quickly to the local fluid velocity. To confirm this, we calculated the slip velocity between the two phases $u_{\text {slip }}=\left|\mathbf{u}_{p}-\mathbf{u}_{f}\right|$ where $\mathbf{u}_{p}$ and $\mathbf{u}_{f}$ are the instantaneous particle and fluid velocities, respectively. To determine the slip velocity, we used a methodology similar to that proposed by Augier et al. [35] and Derksen [36]. For every pair of images, each particle lies in a correlation box sufficiently large to contain both the particle and the minimum amount of tracer necessary for the estimation of the fluid velocity. Employing the PTV and PIV algorithms, one can then measure both the velocity of the particle and that of the surrounding fluid, respectively. From it, we conclude that, for all the instantaneous velocity fields considered, almost all of the spheres have $\left|\mathbf{u}_{p}-\mathbf{u}_{f}\right| /\left|\mathbf{u}_{f}\right| \ll 1$, with the maximum value of $\left|\mathbf{u}_{p}-\mathbf{u}_{f}\right| /\left|\mathbf{u}_{f}\right|$ never exceeding $5 \%$.

Figure 5(b) shows the contour plots of the averaged solid volume fraction, $\langle C\rangle$, and the tangential component of the vorticity, $\omega_{\theta}$, for the GWZ-N mixture at $\mathrm{Re}=65$. The solid volume fraction 
distributions in the contour plot were determined by assigning a " 1 " to pixels that fall inside a detected circle [see Fig. 3(c)] and " 0 " elsewhere. Averaging the pixel values over 50 images then provides an estimate for the average local solids volume fraction field, $\langle C\rangle$, shown in Fig. 5(b). As we can see, the solids are uniformly dispersed up to a minimum height of $z / T=0.45$. The steadystate solids volume fraction field, $\langle C\rangle$, in Fig. 5(b) is reached via two stages. The solid particles are initially placed at the surface of the stationary denser liquid. If the combined buoyancy and surface tension forces are greater than the gravitational force, the particles remain at the surface. Once the impeller is turned on and the fluid starts moving, additional fluid-particle interaction forces emerge, and in particular the drag force. This has to overcome the buoyancy and surface tension forces to draw the particles away from the surface into the liquid bulk in the first stage. The second stage involves the distribution of the solids in the tank owing to the flow. Particles are drawn into the liquid bulk at an impeller speed of $N=700 \mathrm{rpm}$. At this speed, the flow becomes steady while the particles are still on the liquid surface (see Fig. 4). When the particles enter the flow domain, their velocities relax rapidly to the local fluid velocities and follow the fluid pathlines of the large vortical motions above the top impeller, where they remain confined. Also, it can be seen in Fig. 5(a) that some solids follow the path lines of the vortex immediately below the top impeller; this may happen because, near the impeller tip, when the fluid hits the wall, it can be ejected axially upwards or downwards. However, the particles never reach the bottom of the tank due to the segregation of the flow field produced by the dual blade impeller [Fig. 5(a), left plot].

No clustering of the particles in the core of the vortices was observed in this case [see Fig. 5(b)], in contrast to the findings of Wang et al. [20], who reported particle clustering in the core of the vortices for a Newtonian ambient fluid and particles with Stokes number St $\ll 1$. Wang et al. [20] found that particle clustering can take a long time to occur (up to 30 minutes) for large particles $\left(d_{p}=2.8 \mathrm{~mm}\right)$. However, even after $30 \mathrm{~min}$, we did not observe any clustering in the core of the vortices. Since the particles closely follow the fluid pathlines and no clustering is observed in the vessel for the Newtonian ambient fluid, for the rest of the study we will assume that the role of inertia on the trajectory of the particles is negligible.

We then investigated the effects of the viscoelastic solution with xanthan gum (GWZ-NN) on the distribution of the suspended solids. Figure 6 shows the averaged solid volume fraction and vorticity contour plots at steady state for two different Reynolds numbers; also shown are the corresponding averaged axial solid volume fraction profiles, $\langle C\rangle^{*}$. The quantity $\langle C\rangle^{*}$, defined as $\langle C(r)\rangle$, is computed over a cross section of the vessel. As we can see from Fig. 6(a), for $\operatorname{Re}=65$ the averaged solid volume fraction maps are radically different from those observed for the Newtonian case. Now, solids accumulate in the three vortical regions of the flow, two immediately above the top impeller blade and one below it. The axial solid volume fraction profiles for two particle volumetric concentrations, $1 \%$ and 2\%, are shown in Fig. 6(a) (right side). For both, the main features of the profiles agree with the three accumulation areas identified in Fig. 6(a) (left side).

When Re increases to 100, the two vortices in the top of the tank merge to form a larger one just above the top impeller blade, while the bottom vortex elongates along the radial direction and shifts closer to the top impeller blade [Fig. 6(b)]. The changes in the flow field are reflected in the averaged solid volume fraction map. The particles now accumulate in the two large vortical regions above and below the top impeller blade. The axial solid volume fraction profile in Fig. 6(b) (right side) shows two main peaks for the two different particle volumetric concentrations investigated. The bottom peak is larger for the larger solid volume fraction. Moreover, for both particle concentrations, more solids are trapped in the top vortex. That is probably because, as the solids are drawn into the fluid, they first encounter the top vortex, so that most particles become entrapped there; only few particles manage to move further down in the vessel, ending up in the vortex below the top impeller. For $\operatorname{Re}=65$, the behavior is similar, but less pronounced, and the averaged solid volume fractions are higher in the vortical structures above the impeller blade compared to that below.

The spatiotemporal distributions of the solids in the tank at the imaging plane for the GWZ-N and GWZ-NN fluids as well as the Shannon entropy $S$ at $\mathrm{Re}=65$ are shown in Figs. 7(a) and 7(b). The supplemental videos [37] show how the solids distribute in the imaging plane over time for both 

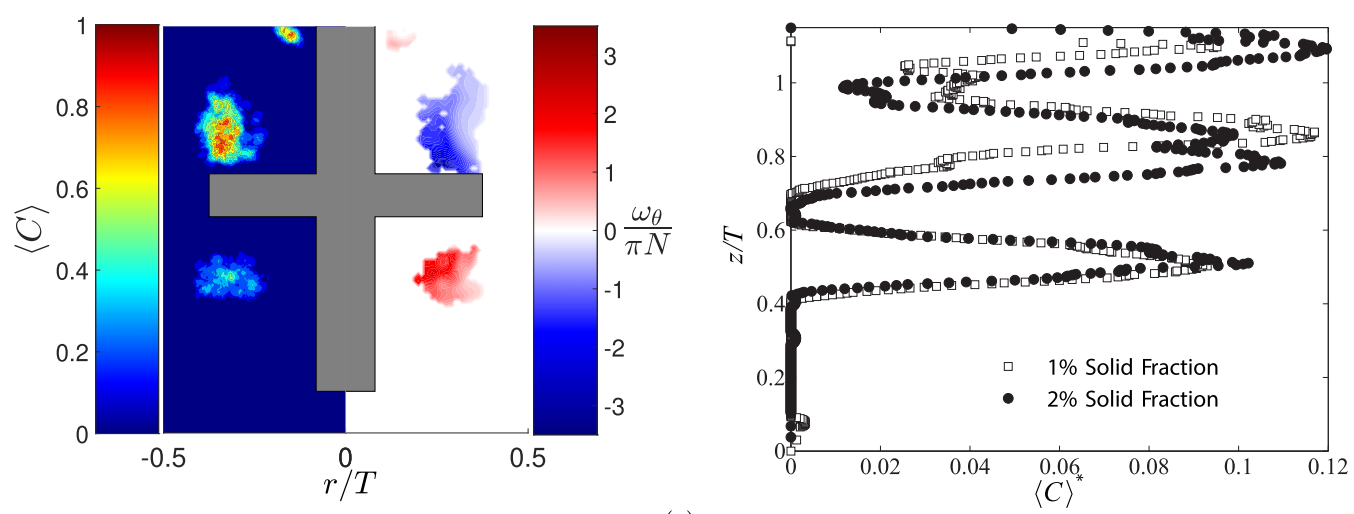

(a)
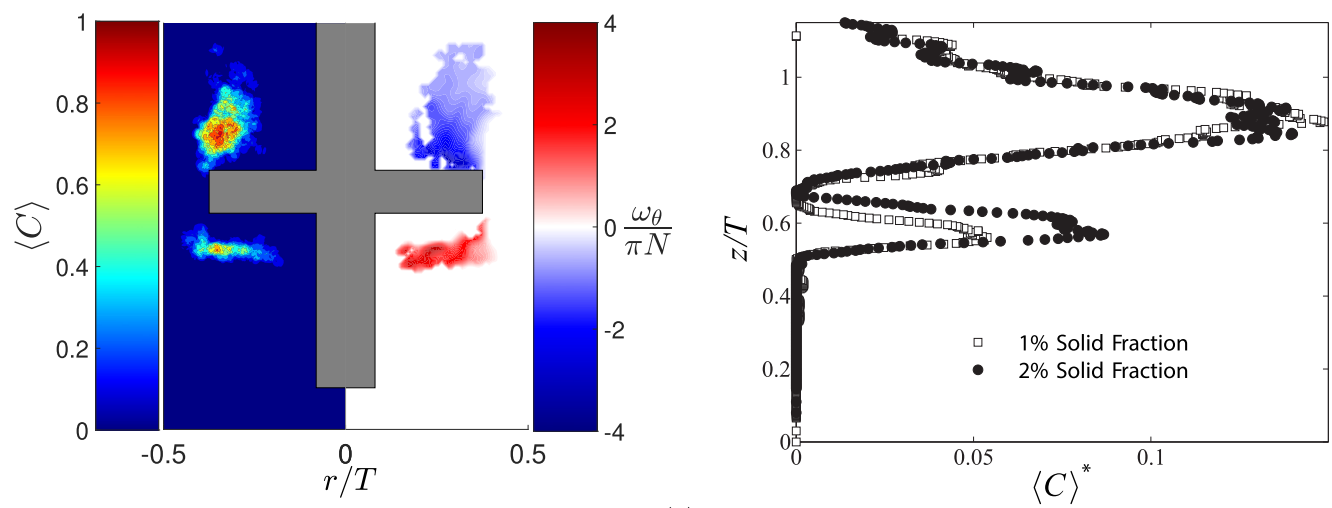

(b)

FIG. 6. Contour plot of the averaged solid volume fraction and dimensionless tangential component of the vorticity, $\omega_{\theta}$, and axial concentration profile, $\langle C\rangle^{*}$, for fluid mixture GWZ-NN and particle volumetric concentrations of $1 \%$ and $2 \%$ at (a) $\operatorname{Re}=65$ and (b) $\operatorname{Re}=100$.

fluids. For GWZ-N [Fig. 7(a)], the Shannon entropy follows a sigmoidal profile with the normalized time $t N . S$ is low initially, when all the particles are at the top of the liquid, as shown in diagram $a$ above Fig. 7(a). As $t N$ increases, $S$ increases monotonically, because the flow draws the particles into the tank along the impeller shaft [see diagram $b$ in Fig. 7(a)]. At $t N=14.6$, where uniform solid mixing has been achieved in the vessel region above the bottom impeller, $S$ plateaus at 0.48 . Similarly, in the non-Newtonian case (GWZ-NN), the Shannon entropy starts from a low initial value, which rapidly increases with time up to a maximum value of around 0.42 . The maximum is lower than the one observed for the GWZ-N mixture, because for fluid GWZ-NN the clustering begins before the solids have time to spread uniformly, almost immediately after the particles are drawn in the vessel; this means that the solids occupy a smaller region of the flow domain [see diagram $b$ above Fig. 7(b)], which results in a lower value of $S$. As $t N$ increases, the Shannon entropy reduces, nearly plateauing at a value of 0.22 with a fluctuation of about $\pm 5 \%$. The decrease of $S$ reflects the reduction in the area of the image occupied by the particles, as shown in diagram $c$ above Fig. 7(b). In stirred tanks, such clustering can affect adversely many process operations that depend on the homogeneous distribution of the solids, including catalysis and mass transfer.

\section{B. Boger fluids}

From the results above, it was unclear whether the particle migration seen in the xanthan gum mixture was due to the shear thinning or to the elastic behavior of the ambient fluid. For this 

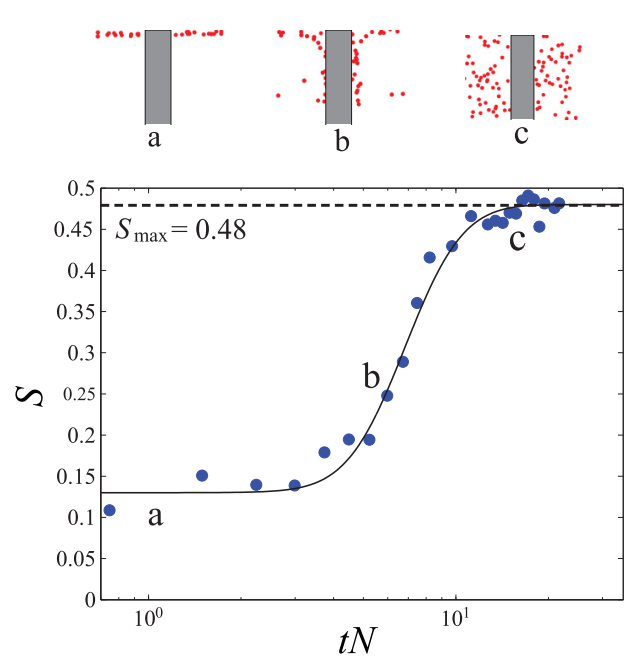

(a)

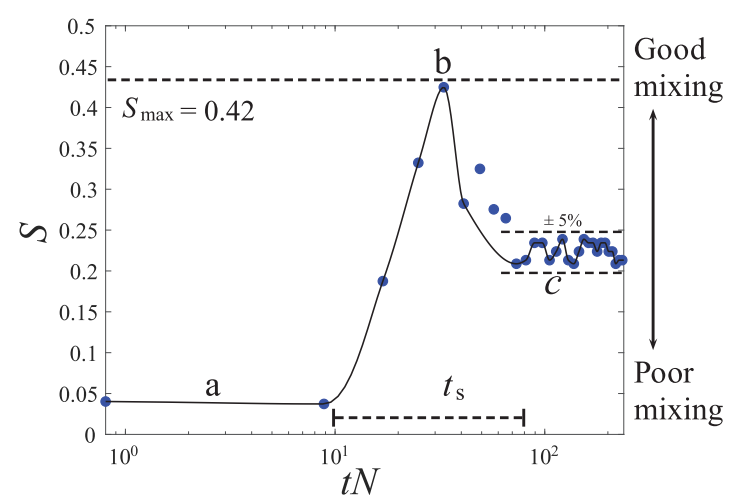

(b)

FIG. 7. Variation of the Shannon entropy, $S$, with increasing $t N$ at $\operatorname{Re}=65$ and particle volumetric concentration of $1 \%$ for (a) GWZ-N; (b) GWZ-NN. See the Supplementary Material [37].

reason, we repeated the experiments with the Boger fluids GW-B1 and GW-B2, where the effect of elasticity can be isolated. In addition, in these fluids the density difference between the liquid and the solid phases is very low $\left(\Delta \rho \approx 60 \mathrm{~kg} / \mathrm{m}^{3}\right)$, a value representative of industrial applications for the separation of solid particles from medium and highly viscous liquids.

Figure 8(a) shows the averaged solid volume fraction (left-half) and vorticity contour plots along with the corresponding velocity vector field (right-half) in the plane of measurement, for the Newtonian $\mathrm{GW}$ mixture $(\mathrm{Wi}=0)$ at $\mathrm{Re}=45$ and particle volumetric concentration of $1 \%$. The contour plot of the averaged solid volume fraction shows that the solids are almost uniformly dispersed throughout the whole measurement plane. Contrary to the GWZ-N case, the solids do reach the bottom of the vessel. This is because with the GW mixture used here the density difference of the two phases is significantly lower, equal to only $60 \mathrm{~kg} / \mathrm{m}^{3}$. This behavior is consistent with the

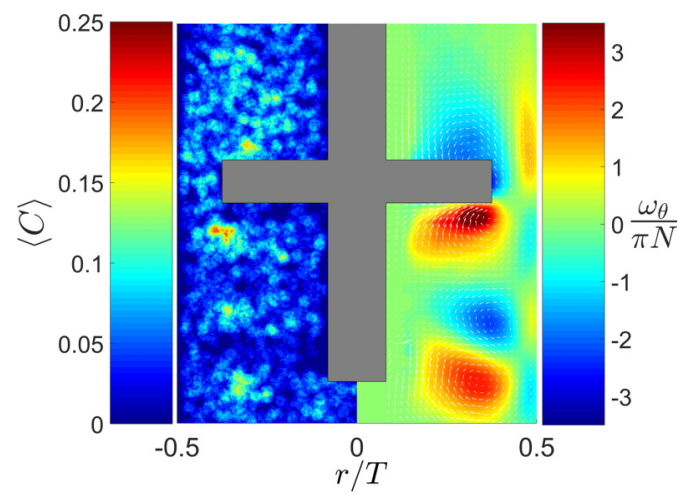

(a)

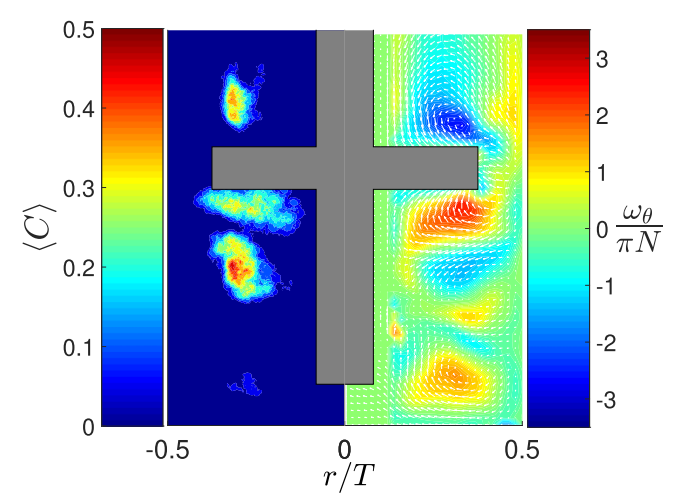

(b)

FIG. 8. Averaged solid volume fraction (left figure) and dimensionless vorticity contour plots along with the dimensionless phase-averaged velocity field (right figure) at $\mathrm{Re}=45$ and particle volumetric concentration of $1 \%$ for fluid mixture: (a) GW (Wi $=0)$ and (b) GW-B2 (Wi $=25)$. 


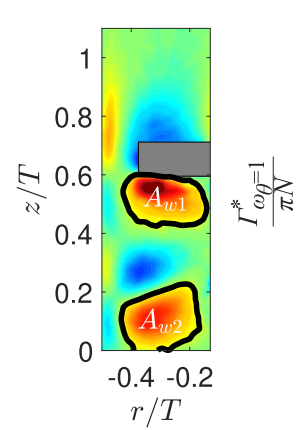

(a)

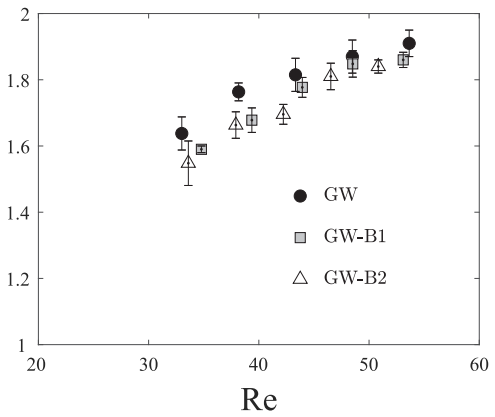

(b)

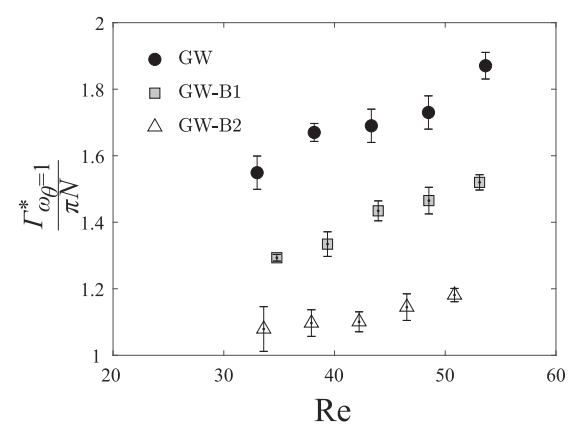

(c)

FIG. 9. (a) Visualization of the area, $A_{\omega i}$, over which the circulation $\Gamma^{*}$ has been estimated (GW mixture, $\mathrm{Wi}=0$ and $\operatorname{Re}=45)$. Variation of the space-averaged dimensionless circulation intensity, $\Gamma_{\omega_{\theta}=1}^{*}$, with increasing $\operatorname{Re}$ for (b) $A_{\omega 1}$, (c) $A_{\omega 2}$.

empirical correlation of Takahashi and Sasaki [38], who showed that the minimum height reached by the suspended phase has the strongest dependence on the density difference between the phases, $\Delta \rho$.

When the PAA polymer is added [Fig. 8(b)], the averaged solid volume fraction map for the particles suspended in the $\mathrm{GW}-\mathrm{B} 2$ mixture $(\mathrm{Wi}=25)$ is similar to that obtained for the suspension in the GWZ-NN mixture (see Fig. 6). The solids have accumulated in each of the vortex cores of the flow. From the right-half of the contour map of Fig. 8(a), one can see that for the GW mixture $(\mathrm{Wi}=0)$ the vortices are formed around the upper and lower sides of the top blade. The intensity of the two vortices is comparable, although they are not symmetrically positioned with respect to the blade center line (i.e., with the upper vortex closer to it). At a phase angle of $\phi=5^{\circ}$, it is still possible to see the vortices generated by the bottom impeller at an angle of $95^{\circ}$ from the bottom leading blade (after a quarter of impeller revolution). Their intensities are still significant and approximately equal to $93 \%$ of the upper blade vortices. The intensity of the upper vortices in the GW-B2 fluid (Wi = 25) shown in Fig. 8(b) (right half) is comparable to that for the GW case $(\mathrm{Wi}=0)$. However, the vortices at the bottom impeller have significantly dissipated after a quarter of a revolution, with intensity values decreased by about $36 \%$ and size shrunk by up to $40 \%$.

These features are summarized in Fig. 9, which shows the circulation intensity, $\Gamma^{*}$, for the different fluid mixtures and varying Reynolds number. $\Gamma^{*}$ is calculated as follows:

$$
\Gamma^{*}=\frac{\int_{A_{\omega i}}\left|\omega_{\theta}\right| d A_{\omega i}}{A_{\omega i}}
$$

in which the integration is carried out over the area $A_{\omega i}$ where the dimensionless vorticity $\omega_{\theta} / \pi N$ is greater than unity. The areas $A_{\omega i}$ over which $\Gamma^{*}$ was estimated are shown in Fig. 9(a) for the case where no particles are suspended in the GW fluid. The variation of $\Gamma^{*}$ for the three mixtures GW, GW-B1, and GW-B2 against Re is shown in Figs. 9(b) and 9(c) for the areas below the top $\left(A_{\omega 1}\right)$ and the bottom $\left(A_{\omega 2}\right)$ impellers, respectively. In both, higher circulation intensity is observed for increasing Re. In the absence of elastic stresses, in the mixture GW, comparable levels of circulation intensity are observed for the vortices confined in the areas $A_{\omega 1}$ and $A_{\omega 2}$. However, for the other two mixtures, GW-B1 and GW-B2, elastic stresses are present and increase with polymer concentration. Around the top impeller, the vortices that were formed on the vertical plane $5^{\circ}$ behind the blade show similar circulation levels for both fluid mixtures. The vortices generated by the bottom impeller were observed at an angle of $95^{\circ}$ from the bottom leading blade (area $A_{\omega 2}$ ) and the suppression by the elastic stresses on the vortices is apparent. For example, at $\mathrm{Re} \approx 50$ the circulation intensity of GW $(\mathrm{Wi}=0)$ is almost $60 \%$ higher than that for GW-B2 $(\mathrm{Wi}=28)$. The suppression of the circulation intensity and the reduction of the vortex size in viscoelastic fluids have previously been reported 


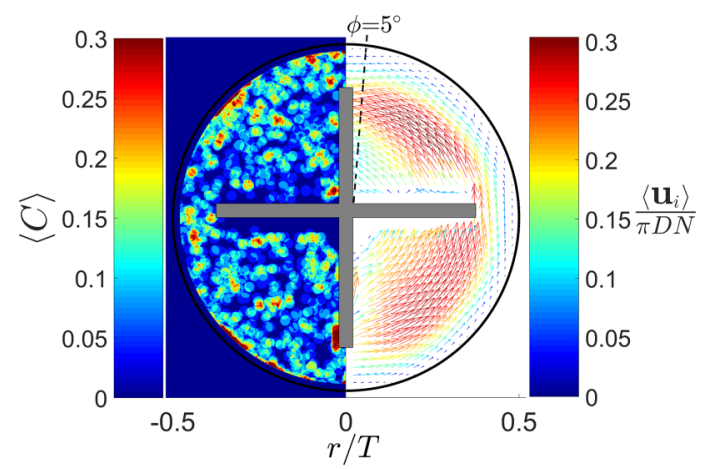

(a)

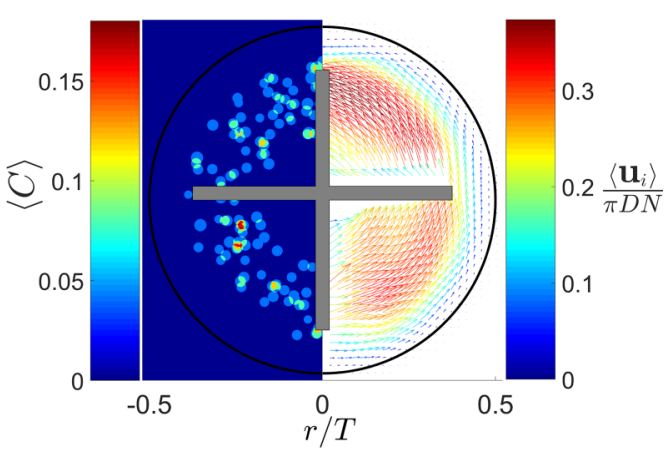

(b)

FIG. 10. Horizontal plane measurements of the averaged solid volume fraction contour map (left half for each figure) and dimensionless velocity field (right half for each figure) at $\operatorname{Re}=45, z / T=0.8$ and particle volumetric concentration of $1 \%$ for fluid mixtures: (a) GW (Wi $=0)$ and (b) GW-B2 (Wi $=25)$.

in the literature and is attributed to the presence of the elastic stresses acting along the circular streamlines of the vortices $[39,40]$.

These results suggest that the solid migration to the core of the vortices and the suppression of the vortices in the flow are caused by the viscoelasticity of the ambient fluid. As reported by D'Avino et al. [41], cross-flow migration of solids has been observed for viscoelastic fluids in the absence of inertia in simple shear and pressure-driven flows in channels with a constant cross section. For almost all the flows examined, the authors found that the solids migrated from regions of high to regions of low shear rate. In the case of a pipe flow, with the fluid flowing in the $z$ direction, the radial migration arises from the combined action of the first normal stress difference $N 1$ and the shear rate gradients. For this simple shear flow, the first normal stress difference is given by $N 1 \equiv \sigma_{z z}-\sigma_{r r}=\beta \dot{\gamma}_{z r}^{2}$, where $\beta$ is a material function [22]. $N 1$ increases with the shear rate [see Fig. 1(b)], and consequently the side of the particle facing the region of high shear rate is subject to a force larger than that acting on the side facing the region of low shear rate. The resultant force is responsible for the migration and is directed from regions of high (close to the pipe wall) to regions of low (close to the pipe center line) shear rates. Solid migration from high to low shear rate regions in viscoelastic fluids has also been reported in a concentric annulus configuration with a rotating inner cylinder [42-44]. In this flow geometry, the inner rotating cylinder produces a shear flow (in the $r$ - $\theta$ plane) which stretches the polymer molecules around the cylinder in the azimuthal direction. This leads to a first normal stress difference $N 1 \equiv \sigma_{\theta \theta}-\sigma_{r r}=\beta \dot{\gamma}_{r \theta}^{2}$ [45], which is larger close to the inner cylinder compared to the outer one. This generates an imbalance in the normal stresses around the particles, a condition that causes a cross-flow migration towards the outer wall, where the shear rate is low.

In the present study, the flow system resembles the concentric annulus flow described above, with the difference that the inner cylinder is equipped with impellers generating vortical flows above and below them. Therefore, to interpret the particle migration observed, measurements need to be taken in the horizontal plane (i.e., in the $r-\theta$ plane). Figures 10(a) and 10(b) exhibit the averaged solid volume fraction contour map and velocity vector fields for the mixtures $\mathrm{GW}(\mathrm{Wi}=0)$ and GW-B2 (Wi $=25)$, at $\mathrm{Re}=45$, for a particle volumetric concentration of $1 \%$ at $z / T=0.8$. The flow fields for both mixtures are typical of rotating fluids with the maximum velocity magnitude near the tip of the impeller and the lowest near the walls $(r / T=0.5)$ and near the edge of the shaft (i.e., $r / T=0.05$ ). The volume fraction contour maps show a uniform distribution of the solids in the plane for GW, while for the GW-B2 fluid the solids tend to localize in the region $r / T \approx 0.2-0.38$.

To understand the accumulation of the particles better, we analyzed the velocity components in the region where the migration occurs. The data in Fig. 11(a) show the radial $\left(u_{r}\right)$, tangential 


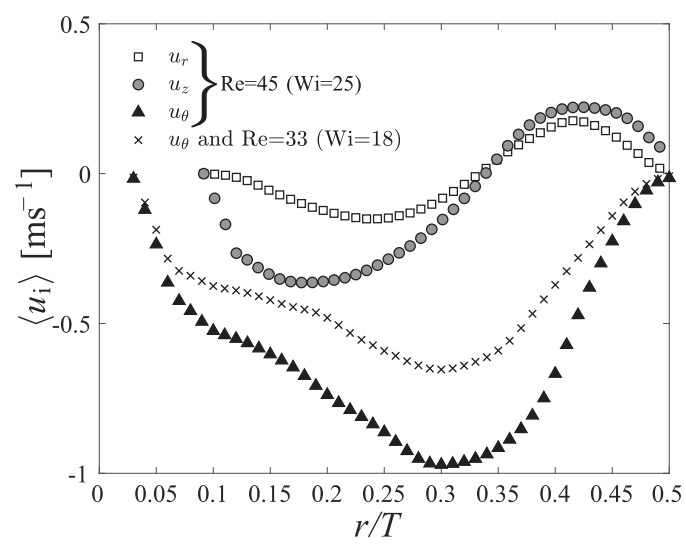

(a)

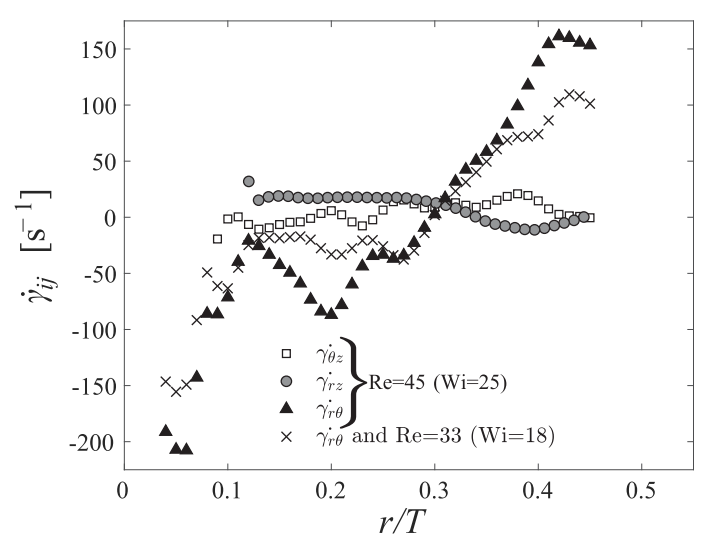

(b)

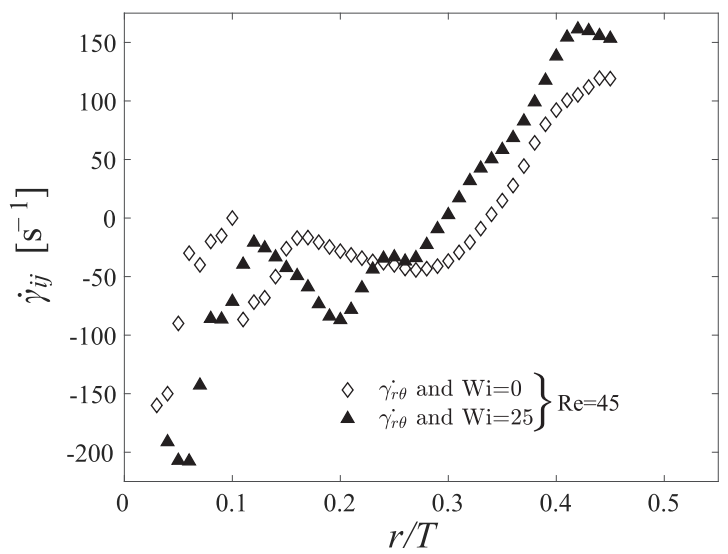

(c)

FIG. 11. Comparison of the radial profiles of the phase-resolved: (a) radial $\left(u_{r}\right)$, tangential $\left(u_{\theta}\right)$, and axial $\left(u_{z}\right)$ velocity profiles; (b) shear rates, $\dot{\gamma}_{\theta z}, \dot{\gamma}_{r z}$, and $\dot{\gamma}_{r \theta}$; obtained from vertical and horizontal plane measurements for the fluid mixture GW-B2 at $z / T=0.8$; (c) shear rate, $\dot{\gamma}_{r \theta}$, for the fluid mixtures GW $(\mathrm{Wi}=0)$ and $\mathrm{GW}-\mathrm{B} 2(\mathrm{Wi}=25)$ for $\mathrm{Re}=45$ at $z / T=0.8$.

$\left(u_{\theta}\right)$, and axial $\left(u_{z}\right)$ velocity component profiles obtained for the mixture GW-B2 at $\phi=5^{\circ}$ and $z / T=0.8$ above the top impeller. For $\mathrm{Re}=45(\mathrm{Wi}=25), u_{\theta}$ exhibits a parabolic-like profile with a maximum absolute value of $1 \mathrm{~m} / \mathrm{s}$ at $r / T=0.33$, which corresponds to the core of the vortex. The maximum absolute value of $u_{\theta}$ is an order of magnitude greater than the maximum absolute value of $u_{r}$ and almost four times that of $u_{z}$. For the same fluid mixture a similar $u_{\theta}$ profile is obtained at $\operatorname{Re}=33(\mathrm{Wi}=18)$, where the maximum absolute value is $0.65 \mathrm{~m} / \mathrm{s}$ and the slope of the profile is less steep than that obtained at $\mathrm{Re}=45(\mathrm{Wi}=25)$. We also calculated the shear rates to identify high shear zones which directly affect the direction of the cross-flow particle migration in the vessel. In Fig. 11(b) the shear rates, $\dot{\gamma}_{\theta z}=\left(\frac{\partial\left\langle u_{\theta}\right\rangle}{\partial z}+\frac{1}{r} \frac{\partial\left\langle u_{z}\right\rangle}{\partial \theta}\right), \dot{\gamma}_{r z}=\left(\frac{\partial\left\langle u_{r}\right\rangle}{\partial z}+\frac{\partial\left\langle u_{z}\right\rangle}{\partial r}\right)$, and $\dot{\gamma}_{r \theta}=\left[\frac{1}{r} \frac{\partial\left\langle u_{r}\right\rangle}{\partial \theta}+r \frac{\partial}{\partial r}\left(\frac{\left\langle u_{\theta}\right\rangle}{r}\right)\right]$, are plotted against the radial coordinate for the same location $z / T=0.8$. The dominant shear rate is clearly $\dot{\gamma}_{r \theta}$, which is an order of magnitude greater than $\dot{\gamma}_{\theta z}$ and $\dot{\gamma}_{r z}$. This indicates that the primary flow in the tank is a pure shear flow in the $r-\theta$ plane where the term $r \frac{\partial}{\partial r}\left(\frac{\left\langle u_{\theta}\right\rangle}{r}\right)$ is dominant. The maximum absolute value of $\dot{\gamma}_{r \theta}$ occurs outside the vortex region, where $\dot{\gamma}_{r \theta}$ exhibits a nearly linear increase, while at the center of the vortex it reaches its minimum 




(a)

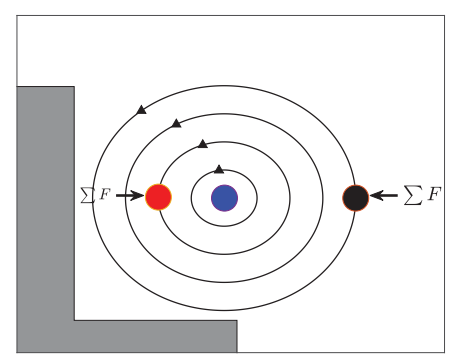

Particle position at:

$$
\begin{aligned}
& t=t_{\mathrm{o}} \\
& t=t_{1} \\
& t=t_{2}
\end{aligned}
$$

(b)

FIG. 12. Schematic diagram of the vortex above the right top impeller and the dynamic of a single particle for (a) Newtonian and (b) purely viscoelastic ambient fluids.

absolute value. This is in agreement with the literature on stirred tank reactors [46], where the shear rate of deformation is lowest at the core of the vortex and highest outside the vortex. At $\operatorname{Re}=33$ $(\mathrm{Wi}=18)$, a similar $\dot{\gamma}_{r \theta}$ profile is obtained, where the absolute values of $\dot{\gamma}_{r \theta}$ are smaller compared to those at $\mathrm{Re}=45(\mathrm{Wi}=25)$. We also compared the dominant shear rate, $\dot{\gamma}_{r \theta}$, profiles obtained for the fluid mixtures $\mathrm{GW}(\mathrm{Wi}=0)$ and $\mathrm{GW}-\mathrm{B} 2(\mathrm{Wi}=25)$ at $\mathrm{Re}=45$ and $z / T=0.8$ as shown in Fig. 11(c). Both profiles show a similar trend where the shear rate has a minimum at the core of the vortex and the absolute value increases further away. Since the flow fields and the shear profiles obtained for both fluid mixtures are similar [see Fig. 8 and Fig. 11(c)], we conclude that the particles migrate radially to the core of the vortices from the combined action of the first normal stress difference $N 1$ (i.e., the fluid elasticity) and the shear rate $\dot{\gamma}_{r \theta}$ gradients.

The particle migration mechanism in the current system is summarized schematically in Fig. 12. As can be seen in Fig. 12(a), in a Newtonian ambient fluid, at different times, a single particle follows the same fluid pathline. This behavior is expected, because the role of inertia on the trajectory of the particle is negligible. Figure 12(b) shows that in a viscoelastic ambient fluid a single particle moves from one vortical pathline to another owing to the imbalance of radial forces acting on it. At the core of the vortex the shear rate has a minimum [see Fig. 11(c)]while further away the absolute value of the shear rate increases. Therefore, the side of the solid (in the radial direction) located far from the core of the vortex faces a region of high shear rate and the other side faces a region of low shear rate. The imbalance of the shear rate causes a difference in the normal force (i.e., a force along in the direction $r$ ) across the particle which pushes it towards the center of the vortex. Once in the middle of the vortex the particle can only move in the tangential direction.

The effects of the different elastic properties on the time required to obtain complete clustering of the solids in the $r-z$ plane was also investigated. To this end, we measured the dimensionless clustering time $t_{s} N$ [see Fig. 6(b)], which represents the time required from the first solid drawdown in the vessel to complete clustering of all the solids in the vessel, at different Wi for the mixtures GW-B1 and GW-B2 as shown in Fig. 13. For the dilute polymeric solutions considered here, the elastic relaxation time $\lambda$ is constant and the Weissenberg number $(\mathrm{Wi}=\lambda N$; see Sec. II C) is varied by increasing the impeller speed, $N$. As expected, for both fluid mixtures $t_{s} N$ decreases exponentially with increasing $\mathrm{Wi}$, because the solids are drawn in the vessel faster. Moreover, higher values of Wi correspond to an increase in the dominant shear rate $\dot{\gamma}_{r \theta}$ [see Fig. 11(b)] and thus an increase in $N 1$, which makes the cross-flow migration faster. It can also be seen that the clustering time $t_{s} N$ for GW-B1 is almost twice that for GW-B2, indicating that doubling the polymer concentration (which increases $N 1$ ) reduces $t_{s} N$ by twofold. For similar Re, the solid clustering times due to viscoelasticity shown in Fig. 13 are at least three orders of magnitude lower than those due to inertia measured by Wang et al. [20]. Finally, it is worth pointing out that the clustering time reported here is not equivalent to the migration time reported in the literature [44] for a single particle, and therefore comparisons between the two times must be made with care. 


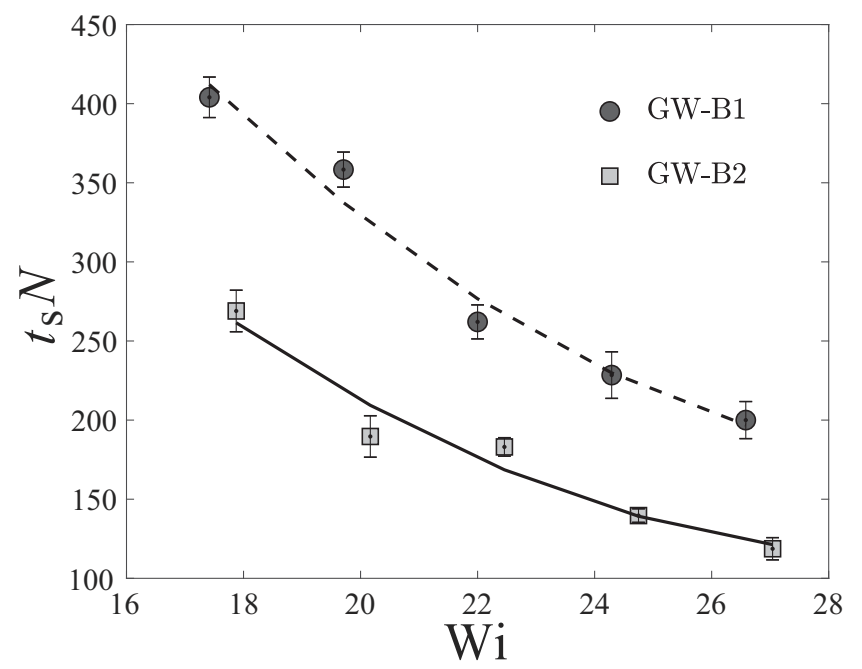

FIG. 13. Variation of the dimensionless solid clustering time, $t_{s} N$, with increasing $\mathrm{Wi}$, solid volume fraction of $1 \%$, and fluid mixtures GW-B1 and GW-B2.

\section{CONCLUSION}

The experimental investigation presented in this paper has shown an interesting phenomenon: particles clustering in the core of vortices via viscoelastic-induced migration. We combined the PLIF, PIV, and PTV techniques to obtain solid concentration, fluid and solid velocity profiles, respectively, in stirred vessels. The experimental data revealed that in a Newtonian ambient fluid the particles disperse uniformly in the plane of measurement and follow the pathlines of the fluid, while in a non-Newtonian ambient fluid they tend to accumulate in the core of the vortices. We employed Boger fluids to isolate the elastic effects, while keeping the density ratio between the phases close to unity to mimic a case in which the separation of the two phases is difficult. We found that the solids did migrate to the core of the vortices when the ambient fluid is purely elastic. We thus examined the effect of the elasticity $(N 1)$ on vortex size and circulation, finding that, in agreement with previous literature, both the vortex size and circulation intensity decrease when the ambient fluid is viscoelastic. We evaluated the clustering time $t_{s} N$ for various values of Wi and for fluids with different polymer concentrations, recording a monotonic decrease of $t_{s} N$ for increasing Wi and polymer concentration. We also found that the clustering time of the solids for viscoelasticity-induced migration was at least three orders of magnitude lower than that observed by Wang et al. [20] for inertia-induced migration.

The particle migration phenomenon observed in this study can be used for separating solid particles from medium and high-viscous liquids. This is particularly beneficial for the cases when the densities of the particles and the ambient liquid are very close and gravity settling cannot be used.

\section{ACKNOWLEDGMENT}

The authors would like to acknowledge GlaxoSmithKline Consumer Healthcare and the EPSRC formulation grant CORAL (EP/N024915/1) for the financial support given to this project.

[1] E. L. Paul, V. A. Atieno-Obeng, and S. M. Kresta, Handbook of Industrial Mixing (Wiley Online Library, New York, 2004). 
[2] R. Chhabra and J. Richardson, Liquid mixing, in Non-Newtonian Flow and Applied Rheology, 2nd ed., edited by R. Chhabra and J. Richardson (Butterworth-Heinemann, Oxford, 2008), pp. 376-461.

[3] A. Gabriele, A. Tsoligkas, I. Kings, and M. Simmons, Use of PIV to measure turbulence modulation in a high throughput stirred vessel with the addition of high Stokes number particles for both up- and down-pumping configurations, Chem. Eng. Sci. 66, 5862 (2011).

[4] J. Nouri and J. Whitelaw, Particle velocity characteristics of dilute to moderately dense suspension flows in stirred reactors, Int. J. Multiphase Flow 18, 21 (1992).

[5] P. Guiraud, J. Costes, and J. Bertrand, Local measurements of fluid and particle velocities in a stirred suspension, Chem. Eng. J. 68, 75 (1997).

[6] J. Derksen, Numerical simulation of solids suspension in a stirred tank, AIChE J. 49, 2700 (2003).

[7] M. Micheletti and M. Yianneskis, Study of fluid velocity characteristics in stirred solid-liquid suspensions with a refractive index matching technique, J. Process Mech. Eng. 218, 191 (2004).

[8] G. Montante, A. Paglianti, and F. Magelli, Analysis of dilute solid-liquid suspensions in turbulent stirred tanks, Chem. Eng. Res. Des. 90, 1448 (2012).

[9] O. Bertrand, B. Blais, F. Bertrand, and L. Fradette, Complementary methods for the determination of the just-suspended speed and suspension state in a viscous solid-liquid mixing system, Chem. Eng. Res. Des. 136, 32 (2018).

[10] S. Ibrahim and A. Nienow, Comparing impeller performance for solid-suspension in the transitional flow regime with Newtonian fluids, Chem. Eng. Res. Des. 77, 721 (1999).

[11] M. Lassaigne, B. Blais, L. Fradette, and F. Bertrand, Experimental investigation of the mixing of viscous liquids and non-dilute concentrations of particles in a stirred tank, Chem. Eng. Res. Des. 108, 55 (2016).

[12] X. Li, C. Yang, G. Zhang, Z.-S. Mao, and H. Zhou, Experimental studies on suspension of solid particles in a low-shear stirred vessel, Chem. Eng. Tech. 34, 1581 (2011).

[13] H. Gong, F. Huang, Z. Li, Z. Gao, and J. Derksen, Mechanisms for drawdown of floating particles in a laminar stirred tank flow, Chem. Eng. J. 346, 340 (2018).

[14] Y. Kawase, K. Shimizu, T. Araki, and T. Shimodaira, Hydrodynamics in three-phase stirred tank reactors with non-Newtonian fluids, Ind. Eng. Chem. Res. 36, 270 (1997).

[15] R. Mollaabbasi and J. M. Najmabad, Experimental investigation and optimization of solid suspension in non-Newtonian liquids at high solid concentration, J. Appl. Fluid Mech. 9, 1907 (2016).

[16] T. Virdung and A. Rasmuson, Measurements of continuous phase velocities in solid-liquid flow at elevated concentrations in a stirred vessel using LDV, Chem. Eng. Res. Des. 85, 193 (2007).

[17] H. Unadkat, C. Rielly, G. Hargrave, and Z. Nagy, Application of fluorescent PIV and digital image analysis to measure turbulence properties of solid-liquid stirred suspensions, Chem. Eng. Res. Des. 87, 573 (2009).

[18] T. Virdung and A. Rasmuson, Solid-liquid flow at dilute concentrations in an axially stirred vessel investigated using particle image velocimetry, Chem. Eng. Commun. 195, 18 (2007).

[19] G. Li, Z. Li, Z. Gao, J. Wang, Y. Bao, and J. Derksen, Particle image velocimetry experiments and direct numerical simulations of solids suspension in transitional stirred tank flow, Chem. Eng. Sci. 191, 288 (2018).

[20] S. Wang, G. Metcalfe, R. L. Stewart, J. Wu, N. Ohmura, X. Feng, and C. Yang, Solid-liquid separation by particle-flow-instability, Energy Env. Sci. 7, 3982 (2014).

[21] M. Cortada-Garcia, W. H. Weheliye, V. Dore, L. Mazzei, and P. Angeli, Computational Fluid Dynamic studies of mixers for highly viscous shear thinning fluids and PIV validation, Chem. Eng. Sci. 179, 133 (2018).

[22] D. F. James, Boger fluids, Annu. Rev. Fluid Mech. 41, 129 (2009).

[23] A. B. Metzner and R. E. Otto, Agitation of non-Newtonian fluids, AIChE J. 3, 3 (1957).

[24] B. H. Zimm, Dynamics of polymer molecules in dilute solution: Viscoelasticity, flow birefringence and dielectric loss, J. Chem. Phys. 24, 269 (1956).

[25] W. M. Abed, R. D. Whalley, D. J. Dennis, and R. J. Poole, Experimental investigation of the impact of elastic turbulence on heat transfer in a serpentine channel, J. Non-Newtonian Fluid Mech. 231, 68 (2016).

[26] F. Del Giudice, S. J. Haward, and A. Q. Shen, Relaxation time of dilute polymer solutions: A microfluidic approach, J. Rheol. 61, 327 (2017). 
[27] F. Del Giudice, G. D’Avino, F. Greco, I. De Santo, P. A. Netti, and P. L. Maffettone, Rheometry-on-a-chip: Measuring the relaxation time of a viscoelastic liquid through particle migration in microchannel flows, Lab Chip 15, 783 (2015).

[28] J. Mark, Polymer Data Handbook, 2nd ed. (Oxford University Press, Oxford, 2009).

[29] V. Tirtaatmadja, G. H. McKinley, and J. J. Cooper-White, Drop formation and breakup of low viscosity elastic fluids: Effects of molecular weight and concentration, Phys. Fluids 18, 043101 (2006).

[30] T. Atherton and D. Kerbyson, Size invariant circle detection, Image Vision Comput. 17, 795 (1999).

[31] W. Brevis, Y. Niño, and G. H. Jirka, Integrating cross-correlation and relaxation algorithms for particle tracking velocimetry, Exp. Fluids 50, 135 (2011).

[32] M. Camesasca, M. Kaufman, and I. Manas-Zloczower, Quantifying fluid mixing with the shannon entropy, Macromol. Theory Sim. 15, 595 (2006).

[33] G. B. Brandani, M. Schor, C. E. MacPhee, H. Grubmüller, U. Zachariae, and D. Marenduzzo, Quantifying disorder through conditional entropy: An application to fluid mixing, PLoS ONE 8, e65617 (2013).

[34] J. Westerweel and F. Scarano, Universal outlier detection for PIV data, Exp. Fluids 39, 1096 (2005).

[35] F. Augier, O. Masbernat, and P. Guiraud, Slip velocity and drag law in a liquid-liquid homogeneous dispersed flow, AIChE J. 49, 2300 (2003).

[36] J. Derksen, Highly resolved simulations of solids suspension in a small mixing tank, AIChE J. 58, 3266 (2012).

[37] See Supplemental Material at http://link.aps.org/supplemental/10.1103/PhysRevFluids.5.063302 for the spatiotemporal distributions of the solids in the tank for both fluids.

[38] K. Takahashi and S. Sasaki, Complete drawdown and dispersion of floating solids in agitated vessel equipped with ordinary impellers, J. Chem. Eng. Jpn. 32, 40 (1999).

[39] P. Sousa, P. Coelho, M. Oliveira, and M. Alves, Laminar flow in three-dimensional square-square expansions, J. Non-Newtonian Fluid Mech. 166, 1033 (2011).

[40] C. Palacios-Morales, C. Barbosa, F. Solorio, and R. Zenit, Negative vortices: The formation of vortex rings with reversed rotation in viscoelastic liquids, Phys. Fluids 27, 051703 (2015).

[41] G. D'Avino, F. Greco, and P. L. Maffettone, Particle migration due to viscoelasticity of the suspending liquid and its relevance in microfluidic devices, Annu. Rev. Fluid Mech. 49, 341 (2017).

[42] A. Karnis and S. G. Mason, Particle motions in sheared suspensions. XIX. Viscoelastic media, Trans. Soc. Rheol. 10, 571 (1966).

[43] B. M. Lormand and R. J. Phillips, Sphere migration in oscillatory Couette flow of a viscoelastic fluid, J. Rheol. 48, 551 (2004).

[44] G. D’Avino, F. Snijkers, R. Pasquino, M. A. Hulsen, F. Greco, P. L. Maffettone, and J. Vermant, Migration of a sphere suspended in viscoelastic liquids in Couette flow: Experiments and simulations, Rheologica Acta 51, 215 (2012).

[45] R. Bird, R. Armstrong, and O. Hassager, Dynamics of Polymeric Liquids, vol. 1: Fluid Mechanics, 2nd ed. (John Wiley \& Sons, New York, 1987).

[46] Y. Bouremel, M. Yianneskis, and A. Ducci, Three-dimensional deformation dynamics of trailing vortex structures in a stirred vessel, Ind. Eng. Chem. Res. 48, 8148 (2009). 